\title{
Genetic Diversity and Pathogenicity of Ralstonia solanacearum Causing Tobacco Bacterial Wilt in China
}

Yanyan Li, The Key Lab of Plant Pathology of Hubei Province, College of Plant Science and Technology, Huazhong Agricultural University, Wuhan, 430070, China; and Tobacco Research Institute of Hubei Province, Wuhan, 430030, China; Ji Feng, Tobacco Research Institute of Hubei Province; Hailong Liu, The Key Lab of Plant Pathology of Hubei Province, College of Plant Science and Technology, Huazhong Agricultural University; Lin Wang, China Tobacco Hubei Industrial Co., Ltd., Wuhan, 430040, China; Tom Hsiang, Environmental Sciences, University of Guelph, Guelph, Ontario, Canada; Xihong Li, Tobacco Research Institute of Hubei Province; and Junbin Huang, The Key Lab of Plant Pathology of Hubei Province, College of Plant Science and Technology, Huazhong Agricultural University

\begin{abstract}
Li, Y., Feng, J., Liu, H., Wang, L., Hsiang, T., Li, X., and Huang, J. 2016. Genetic diversity and pathogenicity of Ralstonia solanacearum causing tobacco bacterial wilt in China. Plant Dis. 100:1288-1296.

Bacterial wilt caused by Ralstonia solanacearum is the most serious soilborne disease of tobacco (Nicotiana tabacum) in China. In this study, 89 strains were collected in 2012 to 2014 from across the four major tobacco-growing areas in China. The strains were identified as phylotype I by multiplex polymerase chain reaction and further divided into seven sequevars based on polymorphisms in the endoglucanase $(\mathrm{egl})$ gene. Among the seven sequevars, four $(15,17,34$, and 44$)$ have been previously described as pathogens of tobacco and two (13 and 14), which are reported here on tobacco, were previously found only on other plants. In addition, a new sequevar named 54 was identified. Strains from

tobacco from different regions showed different levels of genetic diversity based on partial $\mathrm{egl}$ gene sequences. The farther north the distribution, the lower the gene diversity found. Pathogenicity of 27 representative strains was assessed by inoculation onto three tobacco cultivars of varying susceptibility. Through cluster analysis of area under the disease progress curve values, the 27 strains were classified into different pathotypes based on virulence; however, no obvious associations were found between sequevar and pathotype. These results will assist in determining geographical distribution of strains, and provide the foundation for breeding and integrated management programs in China.
\end{abstract}

Bacterial wilt caused by Ralstonia solanacearum is considered the most serious soilborne disease of tobacco (Nicotiana tabacum) (Liu et al. 2013). This disease occurs in many tobacco-growing countries with moist tropical to warm-temperate climates (Denny 2006). In China, bacterial wilt has been gradually expanding, seriously threatening the production of tobacco in different areas. $R$. solanacearum usually enters host roots through wounds or natural openings and colonizes the xylem vessels, leading to stunting and chlorosis; often rapid, unilateral wilting of leaves or stems; vascular browning; or even death (Grimault et al. 1994; Remenant et al. 2011). Therefore, in order to document the invasion of $R$. solanacearum in new areas, it is necessary to have comprehensive research on genetic diversity and pathogenicity.

As a highly diversified pathogen, $R$. solanacearum is described as a species complex (Fegan and Prior 2005). Traditionally, it has been divided into five races based on host range and six biovars based on biochemical properties (Denny 2006). Although this traditional race/biovar classification system is still widely used, it has limitations of being laborious and time consuming (Villa et al. 2005). To overcome these limitations and accurately elucidate the genetic diversity within the $R$. solanacearum species complex, molecular technologies have been used. Polymorphisms in the ribosomal DNA region (Seal et al. 1993, 1999; Weller et al. 2000) and functional genes, including $h r p B$, endoglucanase (egl), and fliC (Gillings et al. 1993; Poussier et al. 2000; Schonfeld et al. 2003), of $R$. solanacearum have been studied by polymerase chain reaction (PCR) restriction fragment length polymorphism and amplified fragment length polymorphism.

Fegan and Prior (2005) proposed a new hierarchical classification scheme to partition strains of $R$. solanacearum into four taxonomic

Corresponding author: J. Huang; E-mail: junbinhuang@mail.hzau.edu.cn

Accepted for publication 27 February 2016.

http://dx.doi.org/10.1094/PDIS-04-15-0384-RE

(C) 2016 The American Phytopathological Society levels: species, phylotype, sequevar, and clone. The classification of phylotypes was determined by phylotype-specific multiplexPCR (Pmx-PCR) and was roughly related to geographic origin (I = Asia, II = America, II = Africa and Indian Ocean, and IV = Indonesia). Phylotypes can be further classified into sequevars based on the sequence analysis of $e g l, h r p B$, and $m u t S$ genes (Fegan and Prior 2005; Wicker et al. 2012; Xu et al. 2009). Finally, clones within sequevars can be determined by genomic DNA fingerprinting using repetitive extragenic palindromic PCR (Fegan 2005; Fegan and Prior 2005). Based on the hierarchical classification scheme, strains of $R$. solanacearum species complex can be clearly distinguished, thereby allowing pathologists to better identify the causal agents, which assists in determining geographical distribution of strains and providing the foundation of a successful breeding and integrated management program (Lewis Ivey et al. 2007; Sanchez Perez et al. 2008).

Although the phylogeny and virulence of tobacco strains have been previously studied in China (Fang et al. 2002; Pan et al. 2012; Xu et al. 2009, 2010), strains in those studies were only from several southern provinces and not completely documented from the whole country. Moreover, previous studies have not reported on the relationship between genetic diversity and virulence of tobacco strains. Therefore, a more detailed study is needed to provide comprehensive and systematic analyses of $R$. solanacearum infecting tobacco in China. In this study, we surveyed the geographical distribution of tobacco bacterial wilt and collected the samples from all tobacco-growing regions of China, and also conducted phylogenetic analyses and examined pathogenicity and the relationships between the strains.

\section{Materials and Methods}

Bacterial isolation and preservation. From 2012 to 2014, plants with wilt symptoms were collected from fields located in tobaccogrowing areas throughout China. This work was done with the cooperation of local growers, local industry, and regional Tobacco Research Institutes. $R$. solanacearum was isolated aseptically from the tubers or roots of wilted plants and streaked onto nutrient agar supplemented with $0.05 \%$ tetrazolium chloride (TZC agar) (Kelman 1954). Plates were 
incubated for 3 to 5 days at $28 \pm 2{ }^{\circ} \mathrm{C}$. Colonies with a typical $R$. solanacearum phenotype (single slimy, milky colonies with a pink center) were subcultured onto TZC medium and purified for further study. All strains were maintained as suspensions in sterile distilled water at room temperature or in long-term storage in autoclaved $30 \%$ glycerol solution at $-80^{\circ} \mathrm{C}$ and were revived by plating on TZC agar.

Biovar determination. Biovars were determined as described previously (Hayward 1964). Freshly cultured $R$. solanacearum cells were stabbed into soft agar tubes of Hayward's medium containing $1 \%$ filter-sterilized lactose, maltose, cellobiose, mannitol, sorbitol or dulcitol and incubated at $28 \pm 2{ }^{\circ} \mathrm{C}$ for 14 days. Each test was repeated three times and fresh media were used as negative controls. The culture medium changed from green to yellow in positive cultures, which indicated the ability of strains to oxidize the amended substance.

DNA extraction and phylotype identification. Total DNA of each strain was extracted using the Mini BEST Bacterial Genomic DNA Extraction Kit (ver. 3.0; TaKaRa Bio Inc., Dalian, China), according to the manufacturer's instructions. Following Fegan and Prior (2005), phylotype identification was conducted for 95 strains. Pmx-PCR was carried out in $25-\mu l$ reaction mixtures containing $2.5 \mu \mathrm{l}$ of $10 \times \mathrm{PCR}$ buffer, $2 \mathrm{U}$ of $\mathrm{Taq}$ polymerase, $1.5 \mathrm{mM} \mathrm{MgCl}_{2}$, $200 \mu \mathrm{M}$ each dNTP, 4 pmol each of species-specific primers 759 (5'-GTCGCCGTCAACTCACTTTCC-3') and 760 (5'-GTCGCCGT CAGCAATGCGGAATCG-3') (Opina et al. 1997), 6 pmol each of phylotype-specific primers (Nmult21: 1F, Nmult21: 2F, Nmult23: AF, Nmult22: InF, or Nmult22: RR) (Fegan and Prior 2005), $50 \mathrm{ng}$ of DNA template, and deionized water. The amplifications were performed as follows: an initial denaturation step at $96^{\circ} \mathrm{C}$ for $5 \mathrm{~min}$; followed by 30 cycles of denaturation at $94^{\circ} \mathrm{C}$ for $15 \mathrm{~s}$, annealing at $59^{\circ} \mathrm{C}$ for $30 \mathrm{~s}$, and extension at $72^{\circ} \mathrm{C}$ for $30 \mathrm{~s}$; with a final extension step at $72^{\circ} \mathrm{C}$ for $10 \mathrm{~min}$. Aliquots $(5 \mu \mathrm{l})$ of each reaction mixture were examined by electrophoresis through $2 \%$ agarose gels in Tris-borate-EDTA (TBE) buffer. Bands were visualized with UV light after ethidium bromide staining.

DNA sequencing of partial $\boldsymbol{e g} \boldsymbol{l}$ gene. Sequevar determination was done by sequencing of the partial $e g l$ gene. PCR amplification of a 750-bp region of this gene was performed using the primer pair Endo-F (5'-ATGCATGCCGCTGGTCGCCGC-3') and Endo-R (5'GCGTTGCCCGGCACGAACACC-3') (Poussier et al. 2000). The reaction mixture (total volume of $25 \mu \mathrm{l}$ ) contained $12.5 \mu \mathrm{l}$ of $2 \times$ Es Taq MasterMix (Beijing ComWin Biotech Co., Ltd., Beijing, China), $10 \mu \mathrm{M}$ each primer, $50 \mathrm{ng}$ of DNA as template, and deionized water. PCR was performed using the following program: initial denaturation at $96^{\circ} \mathrm{C}$ for $9 \mathrm{~min}$; followed by 30 cycles of $95^{\circ} \mathrm{C}$ for $1 \mathrm{~min}, 70^{\circ} \mathrm{C}$ for $1 \mathrm{~min}$, and $72^{\circ} \mathrm{C}$ for $2 \mathrm{~min}$; with a 10 -min final extension at $72^{\circ} \mathrm{C}$ and a final hold at $4^{\circ} \mathrm{C}$.

Aliquots $(5 \mu \mathrm{l})$ of the reaction mixtures were examined by electrophoresis through $1.5 \%$ agarose gel in TBE buffer. Bands were visualized with UV light after ethidium bromide staining. PCR products were purified and sequenced (TsingKe Biological Technology Co., Ltd., Wuhan, China).

Sequence analysis and construction of phylogenetic trees. The partial sequences of the $e g l$ gene from 95 strains were edited with BioEdit 7.2.5 (http://www.mbio.ncsu.edu/BioEdit/bioedit.html) and aligned with ClustalW, version 1.6 (http://www.clustal.org/clustal2/). The sequences were deposited in GenBank (Table 1). All sequences from 95 strains in this study and 31 reference strains (Table 1) distributed among four phylotypes and 25 sequevars from various plant hosts were included in the phylogenetic analysis. Neighbor-joining analysis (Jukes and Cantor 1969) was performed with MEGA, version 6.05 (http://www.megasoftware.net/), with 5,000 bootstrap replicates. Strains with the exact same sequence were reduced to a single representative taxon for analysis but the individual names are all displayed on the tree.

Pathogenicity assays. In order to analyze the virulence difference of strains among the four different tobacco-growing regions, about one-third of total strains (27 strains) randomly selected from different regions were used in pathogenicity testing. The strains were inoculated on three tobacco cultivars ('Hongda', 'K326', and 'Yanyan97'), which are known to vary in susceptibility. To prepare inoculum, bacterial strains were grown overnight in liquid nutrient agar at $28^{\circ} \mathrm{C}$ with shaking at $200 \mathrm{rpm}$. Cells were pelleted by centrifugation, suspended in $0.9 \%$ saline solution, and spectrophotometrically adjusted to an optical density at $600 \mathrm{~nm}=0.1$ (approximately $10^{8}$ $\mathrm{CFU} / \mathrm{ml}$ ).

The inoculation tests were performed according to the lateral root inoculation technique (Cellier and Prior 2010). Seedlings in small plastic pots at the fifth- to sixth-true-leaf stage were inoculated by pouring $10 \mathrm{ml}$ of the bacterial suspension onto the lateral roots, which had been severed along one side with a sterilized blade. The inoculated seedlings (10 seedlings per tobacco cultivar per strain) were then incubated in a greenhouse at $28 \pm 2^{\circ} \mathrm{C}$ and $95 \%$ relative humidity. Symptoms were monitored from 7 to 30 days after inoculation and recorded at 3- to 7-day intervals using the following scale: $0=$ plants without visible symptoms, $1=$ chlorotic spots on stems occasionally or less than half of the leaves wilted on unilateral stems, $3=$ black streak less than half the height of the stem or between half to two-thirds of the leaves wilted on unilateral stems, $5=$ black streak over half the length of the stem but not reaching the top of the stem or more than two-thirds of the leaves wilted on unilateral stems, $7=$ black streak reaching the top of the stem or all leaves wilted, and $9=$ plant dead. Based on the number of plants in each rating scale, disease index $(\mathrm{DI})$ was calculated as DI $=100 \times(n 1 \times 1+n 2 \times 3+$ $n 3 \times 5+n 4 \times 7+n 5 \times 9) /[(n 0+n 1+n 2+n 3+n 4+n 5) \times 9]$, where $n 0, n 1, n 2, n 3, n 4$, and $n 5$ represent the number of plants with symptoms of $0,1,3,5,7$, and 9 , respectively. DI ranged from 0 (no disease) to 100 (dead).

The resistance reaction of the three tobacco cultivars to each strain was evaluated at 30 days after inoculation as follows: DI values of (i) $0=$ highly resistant (HR); (ii) 0.1 to $20=$ resistant (R); (iii) 20.1 to $40=$ moderately resistant (MR); (iv) 40.1 to $60=$ moderately susceptible (MS); (v) 60.1 to $80=$ susceptible (S), and (vi) 80.1 to $100=$ highly susceptible (HS).

Area under the disease progress curve (AUDPC) was calculated by the trapezoidal integration of the DI from 7 to 30 days after inoculation as follows:

$$
A U D P C=\sum_{i=1}^{n-1}\left[\frac{X_{i+1}+X_{i}}{2}\right]\left(t_{i+1}-t_{i}\right)
$$

where $X_{i}$ is the DI at the $i$ th observation, $n$ is the total number of the observation, and $\left(t_{i+1}-t_{\mathrm{i}}\right)$ is the time interval in days between two consecutive evaluations (Das et al. 1992; Simko and Piepho 2012). Based on the AUDPC values of three cultivars, cluster analysis was conducted using SPSS, version 19.0 (http://www-01.ibm.com/ software/analytics/spss/), hierarchical cluster analysis to classify the 27 strains into different virulence types, and a dendrogram was produced using SPSS to reflect these relationships. AUDPC values of the three cultivars were subjected to analysis of variance in SPSS. Where significant treatment effects were found $(P \leq 0.05)$, means were separated by the test of least significant difference $(P=0.05)$.

\section{Results}

Disease distribution. There are five tobacco-growing areas in China, including southwestern China (Yunnan, Sichuan, Guizhou, and Guangxi provinces), southeastern China (Fujian, Anhui, Zhejiang, Guangdong, and Jiangxi provinces), the midbasin region of the Yangtze River (Hubei, Hunan, and Chongqing provinces), the lower Yellow/Huai River region (Henan, Shandong, and Shanxi provinces), and northern China (Liaoning, Heilongjiang, and Jilin provinces) (Fig. 1A). In this study, the geographical distribution of tobacco bacterial wilt was surveyed and the samples were collected from all five regions during 2012 to 2014 in China. In all, 89 strains from tobacco ( 85 from flue-cured tobacco, 1 from burley tobacco, and 3 from oriental tobacco) as well as 4 from potato, 1 from tomato, and 1 from peanut were obtained and subjected to various tests. $R$. solanacearum infecting tobacco was found in all regions except for northern China (Fig. 1B). Detailed information on the 89 strains from tobacco and 6 strains from other hosts is presented in Table 1. 
Biovar characteristics. All 89 strains from tobacco were identified as either biovar $3(n=80)$ or biovar $4(n=9)$ based on their ability to oxidize three disaccharides and three hexose alcohols (Table 1). Biovar 4 was only found in southwestern and southeastern China and biovar 3 throughout all four regions. Among the six strains from other hosts, four from potato were identified as biovar 2 or biovar 3 and one from peanut and one from tomato were identified as biovar 3 .

Phylotype analysis. In addition to the expected 280-bp $R$. solanacearum species-complex-specific amplicon generated from the PmxPCR test (Table 1), 93 of the 95 strains generated a single 144-bp phylotype I-specific amplicon and were identified as the Asian Phylotype I. The other two strains from potato each generated a 372-bp amplicon and were identified as the American Phylotype II.
Characterization of partial $\boldsymbol{e g l}$ sequences. Partial $e g l$ gene sequences were obtained for each of the 95 strains to produce the phylogenetic trees (Fig. 2). To properly place these strains, 31 reference strains of $R$. solanacearum representing the known extent of diversity (four phylotypes and 25 sequevars) from different hosts were also included. In the dendrogram depicting phylogenetic positioning, 89 strains from tobacco in China were placed as phylotype I, consistent with the results of the Pmx-PCR analysis. Furthermore, 70 of 89 strains were further grouped into sequevars that have previously been described: sequevars 13, 14, 15, 17, 34, and 44. However, the remaining 19 strains grouped in a single clade with the same variation were placed into a new sequevar that has not yet been described, and has been provisionally named sequevar 54 .

Table 1. Source and relevant information of Ralstonia solanacearum strains used in this study ${ }^{\mathrm{a}}$

\begin{tabular}{|c|c|c|c|c|c|}
\hline Strain $\left(\right.$ GenBank) ${ }^{\mathbf{b}}$ & Host $^{\mathbf{c}}$ & Origin, province & $\mathbf{B v}$ & Phy & Seq \\
\hline \multicolumn{6}{|l|}{ Strains from China $(n=95)$} \\
\hline \multicolumn{6}{|l|}{ (1) Southwestern China } \\
\hline $\begin{array}{l}\text { YNWS1 (KP967674), YNWS2 (KP967675), YNWS3 } \\
\text { (KP967676), YNWS4 (KP967677), YNWS5 } \\
\text { (KP967678), YNWS6 (KP967679), YNWS7 } \\
\text { (KP967680) }\end{array}$ & Tobacco (FC) & Wenshan, Yunnan & 3 & I & 54 \\
\hline GXBS1 (KP967618), GXBS6 (KP967623) & Tobacco (FC) & Baise, Guangxi & 3 & I & 44 \\
\hline GXBS2 (KP967619), GXBS3 (KP967620) & Tobacco (FC) & Baise, Guangxi & 4 & I & 44 \\
\hline GXBS4 (KP967621), GXBS7 (KP967624) & Tobacco (FC) & Baise, Guangxi & 4 & I & 54 \\
\hline GXBS5(KP967622) & Tobacco (FC) & Baise, Guangxi & 3 & I & 54 \\
\hline GXWZ1 (KP967628), GXWZ2 (KP967629) & Tobacco (FC) & Wuzhou, Guangxi & 3 & I & 15 \\
\hline GXHC1 (KP967625), GXHC2 (KP967626) & Tobacco (FC) & Hechi, Guangxi & 4 & I & 17 \\
\hline GXHC3 (KP967627) & Tobacco (FC) & Hechi,Guangxi & 3 & I & 14 \\
\hline $\begin{array}{l}\text { GZBJ1 (KP967630), GZBJ2 (KP967631), GZBJ3 } \\
\text { (KP967632) }\end{array}$ & Tobacco (FC) & Bijie, Guizhou & 3 & I & 17 \\
\hline $\begin{array}{l}\text { GZZY1 (KP967636), GZZY2 (KP967637), GZZY3 } \\
\text { (KP967638) }\end{array}$ & Tobacco (FC) & Zunyi, Guizhou & 3 & I & 17 \\
\hline GZTR1 (KP967633) & Tobacco (FC) & Tongren, Guizhou & 3 & I & 54 \\
\hline GZTR2 (KP967634), GZTR3 (KP967635) & Tobacco (FC) & Tongren, Guizhou & 3 & I & 17 \\
\hline $\begin{array}{l}\text { SCPZH2 (KP967662), SCPZH4 (KP967664), SCPZH6 } \\
\text { (KP967665) }\end{array}$ & Tobacco (FC) & Panzhihua, Sichuan & 3 & I & 44 \\
\hline SCPZH3(KP967663) & Tobacco (FC) & Panzhihua, Sichuan & 4 & I & 44 \\
\hline \multicolumn{6}{|l|}{ (2) Southeastern China } \\
\hline $\begin{array}{l}\text { JXGZ1 (KP967657), JXGZ2 (KP967658), JXGZ3 } \\
\text { (KP967659) }\end{array}$ & Tobacco (FC) & Ganzhou, Jiangxi & 3 & I & 15 \\
\hline JXFZ4(KP967656) & Tobacco (FC) & Fuzhou, Jiangxi & 3 & I & 15 \\
\hline $\begin{array}{l}\text { FJLY1 (KP967602), FJLY2 (KP967603), } \\
\text { FJLY3 (KP967604) }\end{array}$ & Tobacco (FC) & Longyan, Fujian & 3 & I & 15 \\
\hline FJSM1 (KP967608) & Tobacco (FC) & Sanming, Fujian & 4 & I & 34 \\
\hline FJSM2 (KP967609) & Tobacco (FC) & Sanming, Fujian & 3 & I & 34 \\
\hline FJSW3 (KP967610) & Tobacco (FC) & Shaowu, Fujian & 3 & I & 34 \\
\hline $\begin{array}{l}\text { FJNP1 (KP967605), FJNP2 (KP967606), FJNP3 } \\
\text { (KP967607) }\end{array}$ & Tobacco (FC) & Nanping, Fujian & 3 & I & 34 \\
\hline $\begin{array}{l}\text { AHCZ1 (KP967592), AHCZ2 (KP967593), AHCZ3 } \\
\text { (KP967594), AHCZ4 (KP967595) }\end{array}$ & Tobacco (FC) & Chizhou, Anhui & 3 & I & 54 \\
\hline $\begin{array}{l}\text { GDNX1 (KP967611), GDNX3 (KP967613), GDNX8 } \\
\text { (KP967617) }\end{array}$ & Tobacco (FC) & Nanxiong, Guangdong & 3 & I & 13 \\
\hline GDNX2 (KP967612) & Tobacco (FC) & Nanxiong, Guangdong & 4 & I & 15 \\
\hline $\begin{array}{l}\text { GDNX5 (KP967614), GDNX6 (KP967615), GDNX7 } \\
\text { (KP967616) }\end{array}$ & Tobacco (FC) & Nanxiong, Guangdong & 3 & I & 15 \\
\hline ZJSX1 (KP967681), ZJSX2 (KP967682) & Tobacco $(\mathrm{O})$ & Shaoxing, Zhejiang & 3 & I & 15 \\
\hline ZJSX3 (KP967683) & Tobacco $(\mathrm{O})$ & Shaoxing, Zhejiang & 3 & I & 34 \\
\hline \multicolumn{6}{|l|}{ (3) Midbasin region of the Yangtze River } \\
\hline HBJS1 (KP967641) & Tobacco (B) & Jianshi, Hubei & 3 & I & 54 \\
\hline HBBD2 (KP967640) & Tobacco (FC) & Badong, Hubei & 3 & I & 17 \\
\hline HBLC5 (KP967642) & Tobacco (FC) & Lichuan, Hubei & 3 & I & 54 \\
\hline \multirow[t]{2}{*}{ HBXE4 (KP967645) } & Tobacco (FC) & Xuanen, Hubei & 3 & I & 17 \\
\hline & & & \multicolumn{3}{|c|}{ (continued on next page) } \\
\hline
\end{tabular}

a $\mathrm{Bv}=$ biovar, Phy = phylotype, and Seq = sequevar. Sequevar "54" is the new sequevar identified in this study.

b Numbers in parentheses are GenBank accession numbers.

c Tobacco: $\mathrm{FC}=$ flue-cured, $\mathrm{O}=$ Oriental, and $\mathrm{B}=$ burley.

d Strain HA4-1 was provided by Prof. Huilan Chen, Potato Research Team, College of Horticulture \& Forestry Sciences, Huazhong Agricultural University, China.

e Phylotype, sequevar, biovar, host, and origin information for the strains were obtained from previously published literature (Fegan and Prior 2005, 2006; Villa et al. 2005; Wicker et al. 2007). 
Among the seven different sequevars (Fig. 1; Table 2), sequevar $15(33.7 \%)$ in all four areas in China constituted the largest group, followed by sequevar 17 (23.6\%) in the midbasin region of the Yangtze River and southwestern China, and sequevar 54 (21.3\%) in the midbasin region of the Yangtze River, southwestern China, and southeastern China. Sequevars $44(9.0 \%)$ and $14(1.1 \%)$ were found only in southwestern China, and sequevars 34 (7.9\%) and $13(3.4 \%)$ were only isolated from southeastern China.

Among the six strains from other hosts, the one from tomato was placed in sequevar 17 in phylotype I, and the four from potato in sequevar 17 or 18 in phylotype I or sequevar 1 in phylotype II. These sequevars and associations with particular hosts have all previously been reported (Xu et al. 2009). However, one strain from peanut (HA4-1) could not be placed in any of the sequevars, indicating that some $R$. solanacearum strains from peanut in China may belong to an unknown sequevar.

Pathogenicity determination and assessment. Among 89 strains from tobacco, a set of 27 representative strains was selected randomly for pathogenicity testing by inoculation of three tobacco cultivars: Hongda, K326, and Yanyan97. A week after inoculation, slight wilting was usually found on the unilateral stems. The three tobacco cultivars showed differential resistance to bacterial wilt at 30 days after inoculation (Table 3 ). Hongda was the most susceptible, with a final mean DI of $86.4 \pm 18.5$ and AUDPC of $14.3 \pm 4.6$, and

Table 1. (continued from preceding page)

\begin{tabular}{|c|c|c|c|c|c|}
\hline Strain (GenBank) ${ }^{\mathbf{b}}$ & Host $^{c}$ & Origin, province & Bv & Phy & Seq \\
\hline HBLF1 (KP967643) & Tobacco (FC) & Laifeng, Hubei & 3 & $\mathrm{I}$ & 54 \\
\hline HBSYFX2 (KP967644) & Tobacco (FC) & Fangxian, Hubei & 3 & I & 15 \\
\hline $\begin{array}{l}\text { HNFH1 (KP967646), HNFH2 (KP967647), HNFH3 } \\
\text { (KP967648), HNFH4 (KP967649), HNFH5 (KP967650), } \\
\text { HNFH6 (KP967651), HNFH7 (KP967652) }\end{array}$ & Tobacco (FC) & Fenghuang, Hunan & 3 & I & 17 \\
\hline CQQJ1 (KP967596), CQQJ2 (KP967597) & Tobacco (FC) & Qianjiang, Chongqing & 3 & I & 17 \\
\hline CQWL1 (KP967598) & Tobacco (FC) & Wuling, Chongqing & 3 & I & 54 \\
\hline $\begin{array}{l}\text { CQYY1 (KP967599), QYY2 (KP967600), CQYY3 } \\
\text { (KP967601) }\end{array}$ & Tobacco $(\mathrm{FC})$ & Youyang, Chongqing & 3 & I & 15 \\
\hline \multicolumn{6}{|l|}{ (4) Lower Yellow/Huai River region } \\
\hline $\begin{array}{l}\text { SDLY1 (KP967666), SDLY2 (KP967667), SDLY3 } \\
\text { (KP967668), SDLY4 (KP967669), SDLY5 (KP967670), } \\
\text { SDLY6 (KP967671), SDLY7 (KP967672), SDLY8 } \\
\text { (KP967673) }\end{array}$ & Tobacco $(\mathrm{FC})$ & Linyi, Shandong & 3 & I & 15 \\
\hline $\begin{array}{l}\text { HNZMD1 (KP967653), HNZMD2 (KP967654), HNZMD3 } \\
\quad \text { (KP967655) }\end{array}$ & Tobacco $(\mathrm{FC})$ & Zhumadian, Henan & 3 & I & 15 \\
\hline Po43 (KP967661) & Potato & Enshi, Hubei & 3 & I & 18 \\
\hline Po1-1 (KP967660) & Potato & Wuhan, Hubei & 3 & I & 17 \\
\hline HA4-1 (KP967639)d & Peanut & Huanggang, Hubei & 3 & I & 54 \\
\hline Tom1 (KM188491) & Tomato & Wuhan, Hubei & 3 & I & 17 \\
\hline Po42 (KP981387) & Potato & Pengzhou, Sichuan & 2 & II & 1 \\
\hline ES-1 (KP981386) & Potato & Enshi, Hubei & 2 & II & 1 \\
\hline \multicolumn{6}{|l|}{ Reference strains $(n=31)^{\mathrm{e}}$} \\
\hline R292 (AF295255) & Mulberry & China & 5 & I & 12 \\
\hline JT523 (AF295252) & Potato & Reunion Island & 3 & I & 13 \\
\hline CMR134 (EF439740) & Hucklererry & Cameroon & $\ldots$ & $\mathrm{I}$ & 13 \\
\hline Pss81 (FJ561066) & Tomato & Taiwan & 3 & I & 14 \\
\hline Zo4 (FJ561156) & Ginger & Philippines & 4 & I & 14 \\
\hline Pss358 (FJ561056) & Tomato & Taiwan & 3 & I & 15 \\
\hline CFBP765 (EF371810) & Tomato & Japan & $\ldots$ & I & 15 \\
\hline P11 (FJ561068) & Peanut & China & 3 & I & 17 \\
\hline GMI1000 (AL646053) & Tomato & France Guiana & 3 & $\mathrm{I}$ & 18 \\
\hline MAFF211479 (AY464997.1) & Ginger & Japan & 4 & I & 30 \\
\hline JT519 (GU295032.1) & Pelargonium & Reunion Island & $\ldots$ & I & 31 \\
\hline Pss175 (EU407281) & $\ldots$ & Taiwan & $\ldots$ & I & 32 \\
\hline Pe5 (EU407292) & Pepper & Taiwan & 3 & I & 34 \\
\hline Pss219 (FJ561091) & Tomato & China & 3 & I & 34 \\
\hline O3 (FJ561069) & Olive & China & 3 & I & 44 \\
\hline CIP365 (GQ907151.1) & Potato & Philippines & 5 & I & 45 \\
\hline MAD17 (GU295040.1) & Pepper & Madagascar & $\ldots$ & I & 46 \\
\hline GMI8254 (GU295014.1) & Tomato & Indonesia & $\ldots$ & I & 47 \\
\hline M2 (FJ561067) & Mulberry & China & 5 & I & 48 \\
\hline K60 (DQ657614) & Tomato & United States & 1 & II A & 7 \\
\hline IPO1609 (EF371814) & Potato & The Netherlands & 2 & II B & 1 \\
\hline JT516 (AF295258) & Potato & Reunion Island & 2 & II B & 1 \\
\hline MOLK2 (EF371841) & Banana & Philippines & 1 & II B & 3 \\
\hline UW175 (DQ011547) & Banana & Colombia & 1 & II B & 4 \\
\hline CFBP734 (AF295274) & Potato & Madagascar & 1 & III & 19 \\
\hline NCPPB332 (AF295276) & Potato & Zimbabwe & 1 & III & 21 \\
\hline CFBP3059 (AF295270) & Eggplant & Burkina faso & 1 & III & 23 \\
\hline Psi7 (EF371804) & Tomato & Indonesia & 2 & IV & 10 \\
\hline ACH732 (GQ907150) & Tomato & Australia & 2 & IV & 11 \\
\hline $\mathrm{R} 230$ (AF295280) & Banana & Indonesia & $\ldots$ & IV & 10 \\
\hline Ralstonia syzygii R28 (DQ011552) & Syzygium aromaticum & Indonesia & $\ldots$ & IV & 9 \\
\hline
\end{tabular}


it showed MR to only $3.7 \%$ of the strains. The respective values for K326 as an MS cultivar were $65.1 \pm 25.5$ and $10.5 \pm 5.2$, and this cultivar showed MR to $11.1 \%$ and $\mathrm{R}$ to $3.7 \%$ of the strains. Yanyan97 as an R cultivar, with DI $=42.1 \pm 24.7$ and AUDPC $=6.5 \pm 4.2$, showed MR to $33.3 \%$ and $\mathrm{R}$ to $25.9 \%$ of the strains.

By using the cluster analysis based on AUDPC values from the three cultivars, the virulence of the 27 strains was divided into three types, including type $\mathrm{H}$ (high virulence), type $\mathrm{M}$ (moderate virulence), and type L (low virulence) (Fig. 3). Each virulence type was characterized by examining the results of cluster analysis and the resistance reaction of the three tobacco cultivars to each strain (Table 3; Fig. 4). At 30 days after inoculation with the five strains belonging to type $\mathrm{H}$, the mean DI values of the three cultivars were all higher than 80 and AUDPC values were significantly higher $(P<$ $0.01)$ than other virulence types. This indicated that the three cultivars should be considered as S or HS to the five high virulence strains. Against the 12 strains belonging to type M, Hongda and K326 showed MS to HS and Yanyan97 ranged from MR to S. The AUDPC values of the three cultivars were all significantly higher $(P<0.01)$ than those of type L. AUDPC values given by testing the 10 strains belonging to type $\mathrm{L}$ were all significantly lower $(P<$ $0.01)$ than the mean of the two other virulence types. The strains to which Hongda and K326 showed MR and R and Yanyan97 showed $\mathrm{R}$ were all found in this low virulence type.

The analysis of the geographical distribution of different virulence types revealed that low- and moderate-virulence strains were found in all four regions, whereas high-virulence strains were only in the midbasin region of the Yangtze River, southwestern China, and southeastern China (Table 3). This implied that the virulence of strains from the northern parts of the lower Yellow/Huai River region was lower than strains from other regions.

The 27 strains in pathogenicity assays were distributed among all sequevars, except for sequevar 14 . Through the comparative analysis between virulence and sequevars (Table 3; Fig. 3), type $H$ strains were found in sequevars $13,15,17,34$, and 54 ; type $M$ in sequevars 15,17 , and 54; and type $\mathrm{L}$ in sequevars $15,17,34,44$, and 54.

\section{Discussion}

Tobacco bacterial wilt caused by $R$. solanacearum has caused serious economic losses in several southern provinces of China (Liu et al. 2013; Pan et al. 2012; Xu et al. 2009, 2010; Xue et al. 2011). Monoculture (Genin and Boucher 2002) and suitable climatic conditions of southern provinces of China may have positive effects on the development of this soilborne pathogen. Furthermore, due to the movement of plant material and mechanical operations, this vascular disease has also emerged in other areas in China. In this survey, tobacco bacterial wilt was found in four tobacco-growing areas with subtropical, temperate and humid, and subhumid climates but not in the northern China tobacco-growing region with cool monsoon climates. The geographical distribution of $R$. solanacearum of tobacco was in accordance with a previous report (Xu et al. 2009), which described the pathogen distribution on a variety of hosts in China. This result is inconsistent with the viewpoint that high temperature and moisture level are needed for the colonization and spread of this tobacco pathogen.

In previous reports, strains infecting tobacco included biovar 1 in the southeastern United States (Hong et al. 2012); biovar 2N in Japan (Horita and Tsuchiya 2001); biovar 3 in Japan, Korea, and China (Horita and Tsuchiya 2001; Jeong et al. 2007; Xu et al. 2009; Xue et al. 2011); and biovar 4 in China (Xu et al. 2009; Xue et al.

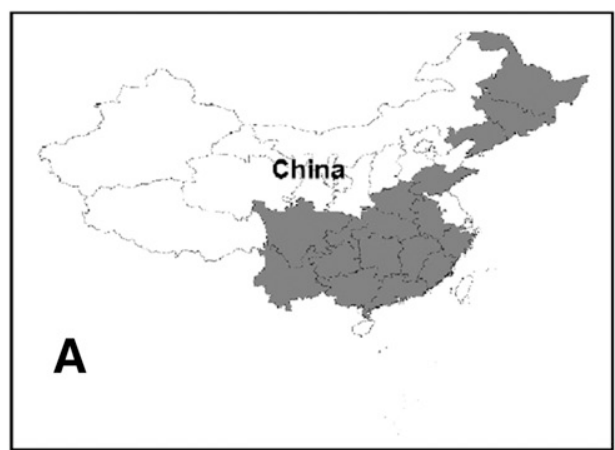

B

$\AA^{N}$

Tobacco-growing areas in China

Southwestern China

Southeastern China

The mid-basin region of the Yangtze River

The lower Yellower/Huai River region

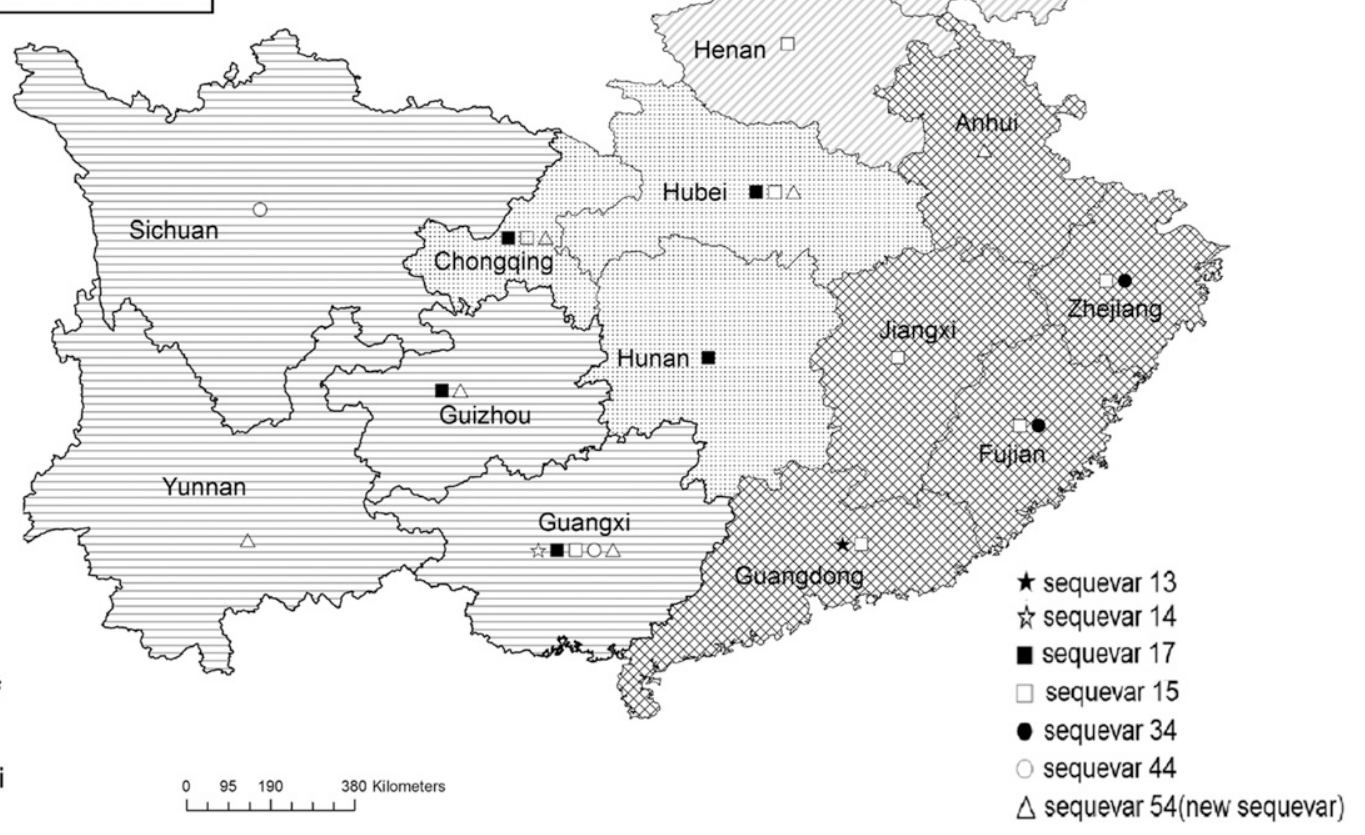

Fig. 1. A, Five tobacco-growing areas and $\mathbf{B}$, distribution of Ralstonia solanacearum infecting tobacco in China. 
2011). In this study, strains characterized as biovars 3 and 4 were also found in China, with the former of much higher prevalence, revealing that biovar 3 from tobacco plants is prevalent in Asia while biovar 4 may be more specific and possibly native to China, especially to the southwest and the southeast.

The results of Pmx-PCR and phylogenetic positioning in this study confirmed that tobacco strains in China belong to Asian Phylotype I, which is consistent with the previous report on tobacco strains from Korea (Jeong et al. 2007). In contrast, tobacco strains from the United States all have been classified as phylotype II (Hong et al. 2012). These results support the association of phylotypes of $R$. solanacearum with geographic origin, as previously stated by Prior and Fegan (2005).

The $e g l$ gene has been widely used to determine the sequevars of R. solanacearum (Fegan and Prior 2005; Prior and Fegan 2005). In the dendrogram depicting the cluster analysis of the partial $e g l$ gene sequences, strains from tobacco in China were partitioned into seven different sequevars, revealing high genetic diversity among the Chinese strains from tobacco. Among the six different reported sequevars, sequevars $15,17,34$, and 44 were previously described on tobacco (Xu et al. 2009), and the other two sequevars (13 and 14), previously described only for other host plants (Xu et al. 2009), are reported here for the first time on tobacco. Following the definition that sequevar can only be used if two or more sequenced strains have similar sequences (Fegan and Prior 2005), the 19 strains which were not placed into previously described sequevars formed their own new sequevar, provisionally named 54 . However, sequevar 54 appears to be closely related to sequevar 44 , as shown on the dendrogram. Future work should verify the relationship between sequevar 54 and other sequevars of $R$. solanacearum by multilocus sequence analysis or whole-genome analyses.

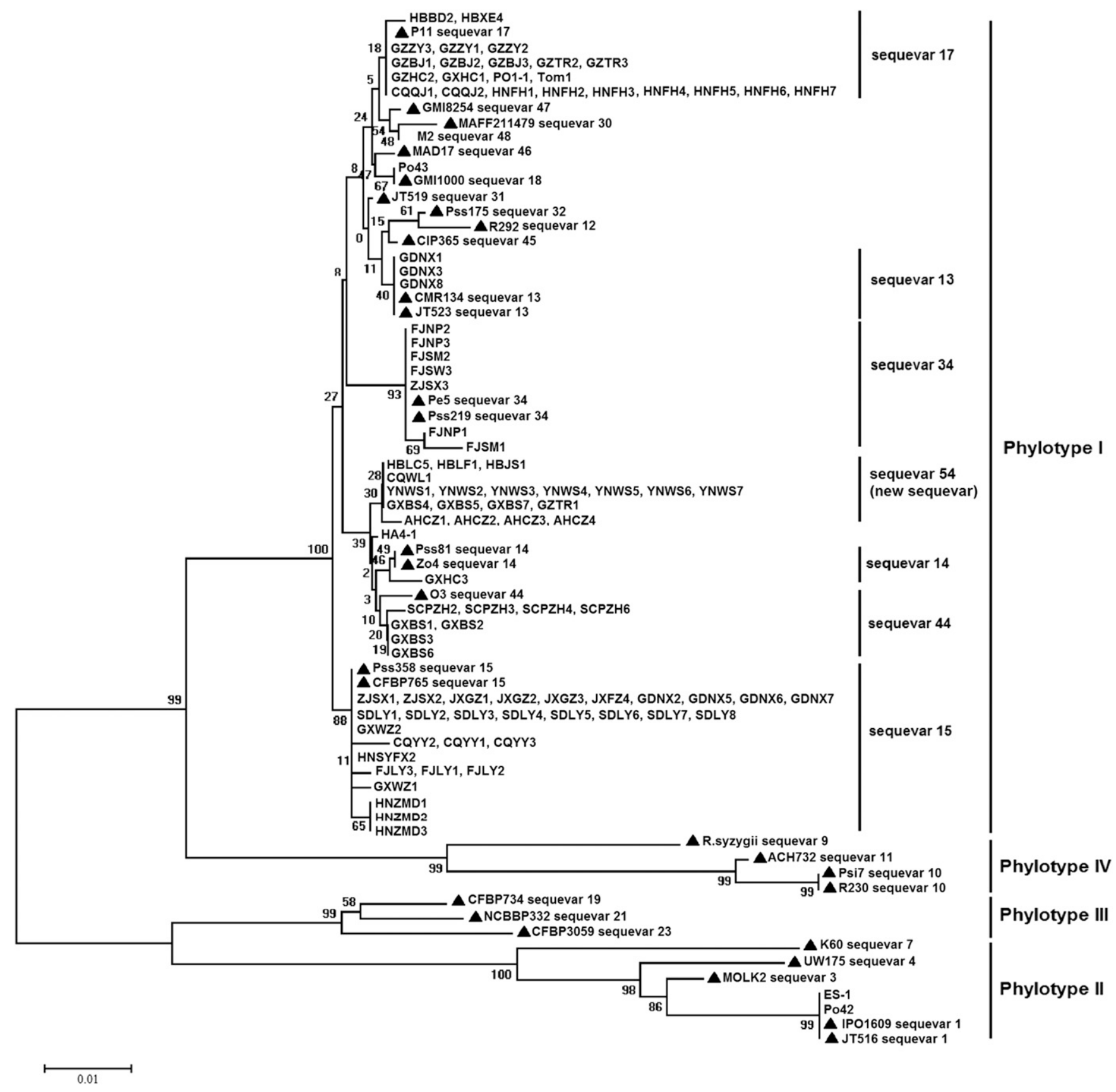

Fig. 2. Dendrogram based on the partial egl gene sequences of 95 Ralstonia solanacearum strains from China and 31 reference strains of sequevars previously deposited in GenBank. The reference strains are represented with $\boldsymbol{\Lambda}$, and all others are strains obtained from China. The dendrogram was generated by MEGA, version 6.05, using neighbor-joining analysis. Values at the branches indicate percent bootstrap support out of 5,000 resamplings. The scale bar represents one nucleotide substitution per 100 nucleotides. 
Previous research (Remenant et al. 2011) using complete genome comparisions has found that phylotypes I and III are most similar, which suggested that phylotypes I and III should be the same species, and phylotype IV and II are two other species. In this study, only phylotype I was found in Chinese tobacco strains of $R$. solanacearum, and the $e g l$ gene showed differences between sequevars within this phylotype, with $0.64 \%$ nonidentical nucleotide sequences between two different sequevars (54 and 44) detected. The low bootstrap values in the phylogenetic tree (Fig. 2) based on the egl gene suggested that this gene may not provide strong support for the separation of sequevars, supporting Hong et al. (2012), who suggested that egl can be misleading for typing strains. However, Ramesh et al. (2014) and Sagar et al. (2014) also observed low bootstrap values in their trees of $R$. solanacearum strains based on egl but continued to use this gene for identification. Further research is needed on sequevar identification and separation.

Table 2. Region in China and sequevar, with number of strains from tobacco in parentheses

\begin{tabular}{ll}
\hline Region $^{\text {a }}$ & \multicolumn{1}{c}{ Sequevar (number of strains) } \\
\hline Southwestern China & $54(11), 44(8), 14(1), 15(2), 17(10)$ \\
Southeastern China & $15(13), 34(7), 54(4), 13(3)$ \\
Midbasin region of the & $54(4), 17(11), 15(4)$ \\
$\quad$ Yangtze River & $15(11)$ \\
Lower Yellow/Huai & \\
River region & \\
\hline
\end{tabular}

${ }^{a}$ Locations of each tobacco-growing region.
Tobacco strains of $R$. solanacearum from different areas in China showed different levels of genetic diversity, as reflected by the different numbers of sequevars observed (Hong et al. 2012). The greatest diversity was found in southwestern and southeastern China, because the strains from the two areas belonged to all seven sequevars found in China, whereas not all sequevars were present in other regions. The next highest diversity was found in the midbasin region of the Yangtze River, with three different sequevars. In contrast, all strains from the lower Yellow/Huai River region were only identified as sequevar 15. Therefore, the farther north the distribution, the lower the genetic diversity observed. The uneven geographic distribution may be due to the differences in temperature adaptation and competitive fitness advantage of $R$. solanacearum strains (Huerta et al. 2015). The temperature in the midbasin region of the Yangtze River and the lower Yellow/Huai River region is slightly lower than that of southwestern and southeastern China and decreases further northward, and some sequevars or groups of strains may be favored by such conditions.

Among the seven sequevars, sequevar 15 constituted the largest group and was widely distributed in all four regions in China, indicating that either sequevar 15 strains have more adaptability to the environment (temperature, humidity, and habitats) than that of other sequevars or they may produce bacteriocins that inhibited growth of other sequevars under temperate conditions (Huerta et al. 2015). Therefore, this sequevar should be prioritized for monitoring in China. Moreover, although strains of four sequevars (sequevars 13, 14,34 , and 44) from tobacco were only found from tobacco in southwestern China and southeastern China, they have been reported from other host plants in the four tobacco-growing regions of China

Table 3. Pathogenicity of Ralstonia solanacearum strains infecting tobacco from China ${ }^{\mathrm{a}}$

\begin{tabular}{|c|c|c|c|c|c|c|c|c|c|c|c|c|}
\hline \multirow[b]{2}{*}{ Strain } & \multicolumn{3}{|c|}{ Hongda } & \multicolumn{3}{|c|}{ K326 } & \multicolumn{3}{|c|}{ Yanyan97 } & \multirow[b]{2}{*}{ Type $^{\text {b }}$} & \multirow[b]{2}{*}{$\mathrm{Seq}^{\mathrm{c}}$} & \multirow[b]{2}{*}{ Area $^{d}$} \\
\hline & DI & $\mathbf{R R}$ & AUDPC & DI & RR & AUDPC & DI & $\mathbf{R R}$ & AUDPC & & & \\
\hline HBLC5 & 100.00 & HS & 19.89 & 94.44 & HS & 17.43 & 86.11 & HS & 15.51 & $\mathrm{H}$ & 54 & WEM \\
\hline JXGZ3 & 100.00 & HS & 21.87 & 100.00 & HS & 16.81 & 100.00 & HS & 14.56 & $\mathrm{H}$ & 15 & WEM \\
\hline FJNP1 & 100.00 & HS & 19.42 & 87.50 & HS & 14.11 & 76.39 & $\mathrm{~S}$ & 14.37 & $\mathrm{H}$ & 34 & WEM \\
\hline GXHC1 & 100.00 & HS & 18.68 & 100.00 & HS & 17.53 & 75.00 & $\mathrm{~S}$ & 11.70 & $\mathrm{H}$ & 17 & WEM \\
\hline GDNX3 & 100.00 & HS & 19.97 & 91.70 & HS & 17.51 & 66.70 & $\mathrm{~S}$ & 10.56 & $\mathrm{H}$ & 13 & WEM \\
\hline GZBJ1 & 87.50 & HS & 16.15 & 87.50 & HS & 14.01 & 37.50 & MR & 5.37 & M & 17 & WEML \\
\hline HNFH1 & 100.00 & HS & 19.36 & 81.94 & HS & 13.49 & 73.61 & $\mathrm{~S}$ & 10.48 & M & 17 & WEML \\
\hline HNZMD3 & 87.50 & HS & 15.56 & 87.50 & HS & 13.38 & 38.89 & MR & 5.60 & M & 15 & WEML \\
\hline YNWS4 & 100.00 & HS & 13.30 & 87.50 & HS & 14.42 & 38.89 & MR & 7.71 & M & 54 & WEML \\
\hline FJLY1 & 100.00 & HS & 14.54 & 84.70 & HS & 15.89 & 38.90 & MR & 4.76 & M & 15 & WEML \\
\hline CQYY1 & 100.00 & HS & 19.54 & 87.50 & HS & 14.31 & 22.22 & MR & 3.20 & M & 15 & WEML \\
\hline HBLF1 & 100.00 & HS & 17.89 & 63.89 & $\mathrm{~S}$ & 13.11 & 56.79 & MS & 8.82 & M & 54 & WEML \\
\hline JXGZ2 & 100.00 & HS & 16.41 & 76.40 & $\mathrm{~S}$ & 12.12 & 58.30 & MS & 8.26 & M & 15 & WEML \\
\hline YNWS1 & 84.70 & HS & 13.95 & 58.33 & MS & 10.92 & 37.50 & MR & 4.42 & M & 54 & WEML \\
\hline SDLY2 & 69.40 & $\mathrm{~S}$ & 14.37 & 68.10 & $\mathrm{~S}$ & 11.87 & 50.00 & MS & 8.86 & M & 15 & WEML \\
\hline HBXE4 & 100.00 & HS & 12.28 & 68.06 & $\mathrm{~S}$ & 10.62 & 31.94 & MR & 6.04 & M & 17 & WEML \\
\hline HNFH4 & 100.00 & HS & 17.98 & 55.56 & MS & 10.99 & 40.28 & MS & 6.95 & M & 17 & WEML \\
\hline HBJS1 & 84.72 & HS & 12.30 & 47.22 & MS & 4.71 & 41.67 & MS & 5.65 & $\mathrm{~L}$ & 54 & WEML \\
\hline HBBD2 & 100.00 & HS & 14.99 & 55.56 & MS & 5.18 & 34.72 & MR & 5.58 & $\mathrm{~L}$ & 17 & WEML \\
\hline AHCZ1 & 75.00 & $\mathrm{~S}$ & 9.57 & 43.10 & MS & 6.79 & 19.40 & $\mathrm{R}$ & 3.32 & $\mathrm{~L}$ & 54 & WEML \\
\hline SDLY5 & 75.00 & $\mathrm{~S}$ & 8.84 & 50.00 & MS & 6.80 & 16.70 & $\mathrm{R}$ & 2.76 & $\mathrm{~L}$ & 15 & WEML \\
\hline AHCZ2 & 84.72 & HS & 8.36 & 50.00 & MS & 6.47 & 9.72 & $\mathrm{R}$ & 1.67 & $\mathrm{~L}$ & 54 & WEML \\
\hline FJSM1 & 76.39 & $\mathrm{~S}$ & 10.40 & 22.22 & MR & 3.47 & 19.40 & $\mathrm{R}$ & 2.11 & $\mathrm{~L}$ & 34 & WEML \\
\hline YNWS2 & 72.20 & $\mathrm{~S}$ & 11.10 & 13.90 & $\mathrm{R}$ & 1.04 & 9.70 & $\mathrm{R}$ & 0.94 & $\mathrm{~L}$ & 54 & WEML \\
\hline CQQJ1 & 56.94 & MS & 8.85 & 23.60 & MR & 3.26 & 19.44 & $\mathrm{R}$ & 2.17 & $\mathrm{~L}$ & 17 & WEML \\
\hline CQWL1 & 44.40 & MS & 5.01 & 47.20 & MS & 3.53 & 27.80 & MR & 3.08 & $\mathrm{~L}$ & 54 & WEML \\
\hline GXBS1 & 33.30 & MR & 6.55 & 25.00 & MR & 3.80 & 9.00 & $\mathrm{R}$ & 0.95 & $\mathrm{~L}$ & 44 & WEML \\
\hline Average & 86.36 & $\ldots$ & 14.34 & 65.13 & $\ldots$ & 10.50 & 42.10 & & 6.50 & $\ldots$ & $\ldots$ & $\ldots$ \\
\hline
\end{tabular}


(Xu et al. 2009). Therefore, in terms of management programs, control of movement of raw tobacco and plant material from these two areas to other areas should be carefully regulated in China.

Through variation analysis in virulence of Chinese strains from tobacco among different regions, the ones from the lower Yellow/Huai River region were found to be less virulent than those from other areas because the high virulence type was not found in this region. In the course of surveying, it was found that the most severe symptoms of tobacco bacterial wilt in the field in this region were less than that in other regions, which might reflect the phenotypic expression of less virulent strains (Zheng et al. 2014). This result indicated an association between virulence and geographic distribution for $R$. solanacearum, which has been found for strains from tomato in Trinidad (Ramsubhag et al. 2012). The stronger soil acidity, deteriorative soil conditions, and unhealthy planting pattern (i.e., tobacco-potato

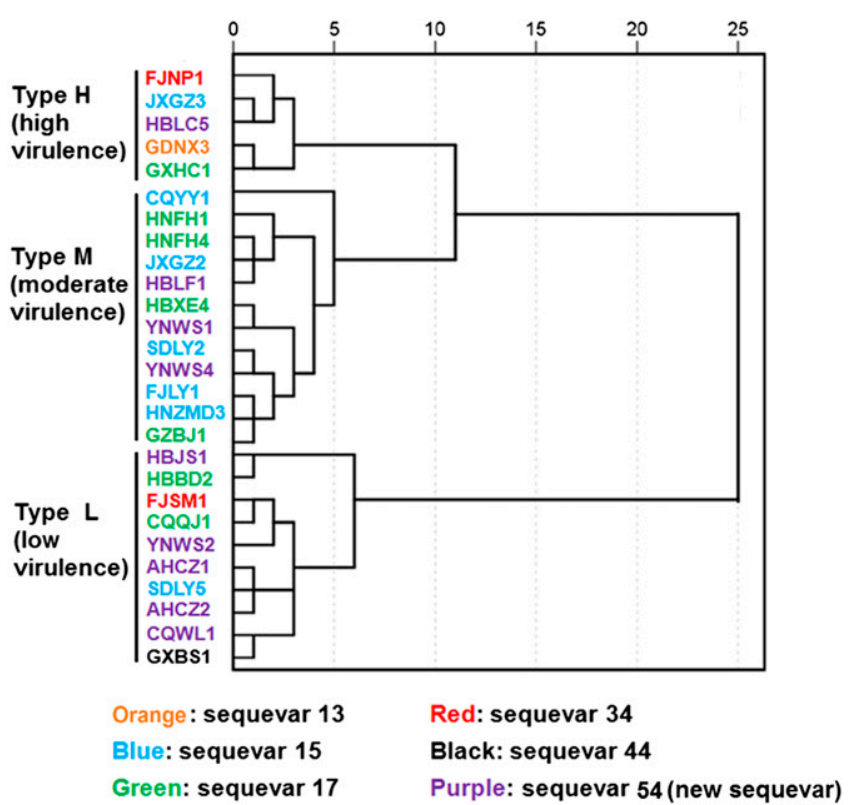

Fig. 3. Dendrogram based on hierarchical cluster analysis of area under the disease progress curve (AUDPC) values for three tobacco cultivars inoculated with 27 representative strains of Ralstonia solanacearum from tobacco. AUDPC values were calculated by the trapezoidal integration of the disease index from 7 to 30 days after inoculation. Strains belonging to different sequevars are marked with different colors.

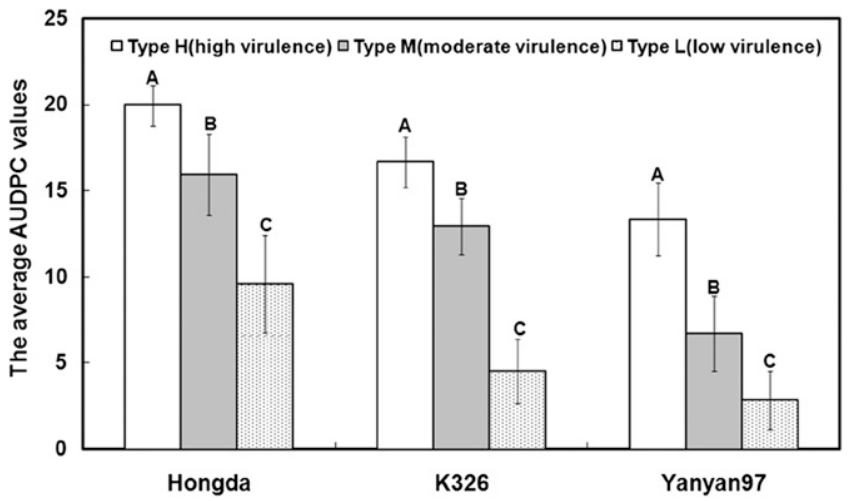

Fig. 4. Mean area under the disease progress curve (AUDPC) values for three tobacco cultivars inoculated with Ralstonia solanacearum strains of different virulence types. Virulence types of strains were divided by hierarchical cluster analysis of AUDPC values for three tobacco cultivars. Types $\mathrm{H}, \mathrm{M}$, and $\mathrm{L}$ contain the high, moderate, and low virulence strains, respectively. Vertical lines at the top of each bar represent the standard error of the mean. AUDPC values of the three cultivars were subjected to analysis of variance in SPSS, and the different capital letters for the same cultivar were significantly different at $P \leq 0.01$ according to Duncan's multiple range test. intercropping) in the other three regions might be the reason for the presence of highly virulent strains. Because only one strain from sequevar 13 and one from 44 was used in the pathogenicity assay, the relationship between sequevars and virulence was definitely analyzed from other sequevars. Sequevars 15,17 , and 54 contained all three virulence types, and sequevar 34 contained both high and low virulence types. Previously, it had been reported that the same phylotype strains could be divided into multiple types based on differences in their pathogenicity (Suga et al. 2013), a result similar to that presented in sequevars in our study. The finding showed that no obvious association was found between the sequevar and virulence, which has also been observed for strains from tomato (Jaunet and Wang 1999). This phenomenon, that the same sequevar strains were represented in multiple pathogenicity types, might be attributed to horizontal gene transfer of virulence factors intersequevar (Liu et al. 2009; Peeters et al. 2013).

This is the first study to characterize strains of $R$. solanacearum of tobacco from across China using phylogenetic and pathogenicity analyses. These results not only will be helpful to the improvement of pathogen-targeted and geographically targeted management practices in China but also will be favorable for tobacco breeding programs. In the virulence assay of this study, compared with the other two tobacco cultivars, Yanyan97 showed strong resistance to bacterial wilt, which has been reported in a previous study (Fang et al. 2002). Therefore, it should be considered as a source of resistance or as a resistant rootstock in grafting or propagation programs. Our results also demonstrated that sequevar 15 strains can adapt themselves to more diverse environments than other sequevar strains. It is assumed that environmentally-induced great adaptation traits are encoded by some genes that are present in the strains belonging to sequevar 15 rather than other sequevars. Alternatively, genes that are present in all sequevars may be induced under slightly changed conditions only in sequevar 15 , resulting in inhibition of the growth of other sequevars which are cohabiting. In future work, a search for genes involved in adaptability to the environment in strains belonging to sequevar 15 or of bacteriocins produced by them under temperate conditions deserves further study, and the northern China tobaccogrowing region should be closely monitored to prevent the introduction of $R$. solanacearum of tobacco, particularly sequevar 15 .

\section{Acknowledgments}

This work was funded by the Hubei Branch of the China National Tobacco Corporation, Grant 027Y2013-002, and China National Tobacco Corporation, Grant 110201502018. We thank all of those who supplied the wilted tobacco plants for isolation and H. Chen (Potato Research Team, College of Horticulture \& Forestry Sciences, Huazhong Agricultural University, China) for providing a strain.

\section{Literature Cited}

Cellier, G., and Prior, P. 2010. Deciphering phenotypic diversity of Ralstonia solanacearum strains pathogenic to potato. Phytopathology 100:1250-1261.

Das, M. K., Rajaram, S., Mundt, C. C., and Kronstad, W. E. 1992. Inheritance of slow-rusting resistance to leaf rust in wheat. Crop Sci. 32:1452-1456.

Denny, T. 2006. Plant pathogenic Ralstonia species. Pages 573-644 in: Plant-Associated Bacteria. S. S. Gnanamanickam, ed. Springer, Dordrecht, The Netherlands.

Fang, S. M., Gu, G., Ji, C. C., Chen, S. H., Zhang, B. S., and Chen, Y. S. 2002 Studies on pathogenic types and distribution of Ralstonia solanacearum in tobacco. Acta Tabacaria Sin. 8:40-43. (In Chinese, with English summary)

Fegan, M. 2005. Bacterial wilt diseases of banana: Evolution and ecology. Pages 379-386 in: Bacterial Wilt Disease and the Ralstonia solanacearum Species Complex. C. Allen, P. Prior, and A. C. Hayward, eds. American Phytopathological Society, St. Paul, MN.

Fegan, M., and Prior, P. 2005. How complex is the "Ralstonia solanacearum Species Complex". Pages 449-461 in: Bacterial Wilt Disease and the Ralstonia solanacearum Species Complex. C. Allen, P. Prior, and A. C. Hayward, eds. American Phytopathological Society, St. Paul, MN.

Fegan, M., and Prior, P. 2006. Diverse members of the Ralstonia solanacearum species complex cause bacterial wilts of banana. Australas. Plant Pathol. 35: 93-101.

Genin, S., and Boucher, C. 2002. Ralstonia solanacearum: Secrets of a major pathogen unveiled by analysis of its genome. Mol. Plant Pathol. 3:111-118.

Gillings, M., Fahy, P., and Davies, C. 1993. Restriction analysis of an amplified polygalacturonase gene fragment differentiates strains of the phytopathogenic bacterium Pseudomonas solanacearum. Lett. Appl. Microbiol. 17:44-48.

Grimault, V., Anais, G., and Prior, P. 1994. Distribution of Pseudomonas solanacearum in the stem tissues of tomato plants with different levels of resistance to bacterial wilt. Plant Pathol. 43:663-668. 
Hayward, A. C. 1964. Characteristics of Pseudomonas solanacearum. J. Appl. Bacteriol. 27:265-277.

Hong, J. C., Norman, D. J., Reed, D. L., Momol, M. T., and Jones, J. B. 2012. Diversity among Ralstonia solanacearum strains isolated from the southeastern United States. Phytopathology 102:924-936.

Horita, M., and Tsuchiya, K. 2001. Genetic diversity of Japanese strains of Ralstonia solanacearum. Phytopathology 91:399-407.

Huerta, A. I., Milling, A., and Allen, C. 2015. Tropical strains of Ralstonia solanacearum outcompete race 3 biovar 2 strains at lowland tropical temperatures. Appl. Environ. Microbiol. 81:3542-3551.

Jaunet, T. X., and Wang, J. F. 1999. Variation in genotype and aggressiveness diversity of Ralstonia solanacearum race 1 isolated from tomato in Taiwan. Phytopathology 89:320-327.

Jeong, Y., Kim, J., Kang, Y., Lee, S., and Hwang, I. 2007. Genetic diversity and distribution of Korean isolates of Ralstonia solanacearum. Plant Dis. 91: 1277-1287.

Jukes, T. H., and Cantor, C. R. 1969. Evolution of protein molecules. Pages 121-132 in: Mammalian Protein Metabolism. H. N. Munro, ed. Academic Press, New York.

Kelman, A. 1954. The relationship of pathogenicity in Pseudomonas solanacearum to colony appearance on a tetrazolium chloride medium. Phytopathology 44: 693-695.

Lewis Ivey, M. L., McSpadden Gardner, B. B., Opina, N., and Miller, S. A. 2007. Diversity of Ralstonia solanacearum infecting eggplant in the Philippines. Phytopathology 97:1467-1475.

Liu, Y. Q., Kanda, A., Yano, K., Kiba, A., Hikichi, Y., Aino, M., Kawaguchi, A., Mizoguchi, S., Nakaho, K., Shiomi, H., Takikawa, Y., and Ohnishi, K. 2009. Molecular typing of Japanese strains of Ralstonia solanacearum in relation to the ability to induce a hypersensitive reaction in tobacco. J. Gen. Plant Pathol. 75:369-380.

Liu, Y. X., Shi, J. X., Feng, Y. G., Yang, X. M., Li, X., and Shen, Q. R. 2013. Tobacco bacterial wilt can be biologically controlled by the application of antagonistic strains in combination with organic fertilizer. Biol. Fertil. Soils 49:447-464.

Opina, N., Tavner, F., Holloway, G., Wang, J. F., Li, T. H., Maghirang, R., Fegan, M., Hayward, A. C., Krishnapillai, V., Hong, W. F., Holloway, B. W., and Timmis, J. N. 1997. A novel method for development of species and strain-specific DNA probes and PCR primers for identifying Burkholderia solanacearum (formerly Pseudomonas solanacearum). Asia Pac. J. Mol. Biol. Biotechnol. 5:19-33.

Pan, Zh. Ch., Xu, J., Gu, G., Wu, W., Xu, J. Sh., Chen, Sh. H., and Feng, J. 2012. Phylogeny of tobacco Ralstonia solanacearum strains from Fujian and Guizhou Provinces. Plant Prot. 38:18-23. (In Chinese, with English summary)

Peeters, N., Carrère, S., Anisimova, M., Plener, L., Cazalé, A.-C., and Genin, S. 2013. Repertoire, unified nomenclature and evolution of the type III effector gene set in the Ralstonia solanacearum species complex. BMC Genomics 14:859.

Poussier, S., Prior, P., Luisetti, J., Hayward, C., and Fegan, M. 2000. Partial sequencing of the $h r p B$ and endoglucanase genes confirms and expands the known diversity within the Ralstonia solanacearum species complex. Syst. Appl. Microbiol. 23:479-486.

Prior, P., and Fegan, M. 2005. Recent development in the phylogeny and classification of Ralstonia solanacearum. Acta Hortic. 695:127-136.

Ramesh, R., Achari, G. A., and Gaitonde, S. 2014. Genetic diversity of Ralstonia solanacearum infecting solanaceous vegetables from India reveals the existence of unknown or newer sequevars of Phylotype I strains. Eur. J. Plant Pathol. 140:543-562.

Ramsubhag, A., Lawrence, D., Cassie, D., Fraser, R., Umaharan, P., Prior, P., and Wicker, E. 2012. Wide genetic diversity of Ralstonia solanacearum strains affecting tomato in Trinidad, West Indies. Plant Pathol. 61:844-857.
Remenant, B., de Cambiaire, J.-C., Cellier, G., Jacobs, J. M., Mangenot, S., Barbe, V., Lajus, A., Vallenet, D., Medigue, C., Fegan, M., Allen, C., and Prior, P. 2011. Ralstonia syzygii, the blood disease bacterium and some Asian $R$ solanacearum strains form a single genomic species despite divergent lifestyles. PLoS One 6:e24356.

Sagar, V., Jeevalatha, A., Mian, S., Chakrabarti, S. K., Gurjar, M. S., Arora, R. K., Sharma, S., Bakade, R. R., and Singh, B. P. 2014. Potato bacterial wilt in India caused by strains of phylotype I, II and IV of Ralstonia solanacearum. Eur. J. Plant Pathol. 138:51-65.

Sanchez Perez, A., Mejia, L., Fegan, M., and Allen, C. 2008. Diversity and distribution of Ralstonia solanacearum strains in Guatemala and rare occurrence of tomato fruit infection. Plant Pathol. 57:320-331.

Schonfeld, J., Heuer, H., van Elsas, J. D., and Smalla, K. 2003. Specific and sensitive detection of Ralstonia solanacearum in soil on the basis of PCR amplification of flic fragments. Appl. Environ. Microbiol. 69:7248-7256.

Seal, S. E., Jackson, L. A., Young, J. P. W., and Daniels, M. J. 1993. Differentiation of Pseudomonas solanacearum, Pseudomonas syzygii, Pseudomonas pickettii and the blood disease bacterium by partial $16 \mathrm{~S}$ rRNA sequencing: Construction of oligonucleotide primers for sensitive detection by polymerase chain reaction. J. Gen. Microbiol. 139:1587-1594.

Seal, S. E., Taghavi, M., Fegan, N., Hayward, A. C., and Fegan, M. 1999 Determination of Ralstonia (Pseudomonas) solanacearum rDNA subgroups by PCR tests. Plant Pathol. 48:115-120.

Simko, I., and Piepho, H.-P. 2012. The area under the disease progress stairs: Calculation, advantage, and application. Phytopathology 102:381-389.

Suga, Y., Horita, M., Umekita, M., Furuya, N., and Tsuchiya, K. 2013. Pathogenic characters of Japanese potato strains of Ralstonia solanacearum. J. Gen. Plant Pathol. 79:110-114.

Villa, J. E., Tsuchiya, K., Horita, M., Natural, M., Opina, N., and Hyakumachi, M 2005. Phylogenetic relationships of Ralstonia solanacearum species complex strains from Asia and other continents based on 16S rDNA, endoglucanase, and $h r p B$ gene sequences. J. Gen. Plant Pathol. 71:39-46.

Weller, S. A., Elphinstone, J. G., Smith, N. G., Boonham, N., and Stead, D. E 2000. Detection of Ralstonia solanacearum strains with a quantitative, multiplex, real-time, fluorogenic PCR (TaqMan) assay. Appl. Environ. Microbiol. 66: 2853-2858.

Wicker, E., Grassart, L., Coranson-Beaudu, R., Mian, D., Guilbaud, C., Fegan, M., and Prior, P. 2007. Ralstonia solanacearum strains from Martinique (French West Indies) exhibiting a new pathogenic potential. Appl. Environ. Microbiol. 73:6790-6801.

Wicker, E., Lefeuvre, P., de Cambiaire, J. C., Lemaire, C., Poussier, S., and Prior, P. 2012. Contrasting recombination patterns and demographic histories of the plant pathogen Ralstonia solanacearum inferred from MLSA. ISME J. 6: 961-974.

Xu, J., Gu, G., Pan, Zh. Ch., Wu, W., Xu, J. Sh., Zhang, H., Chen, Sh. H., and Feng, J. 2010. Phylotype identification and biovar determination of Ralstonia solanacearum strains isolated from Fujian Province. Acta Tabacaria Sin. 16: 66-71. (In Chinese, with English summary)

Xu, J., Pan, Z. C., Prior, P., Xu, J. S., Zhang, Z., Zhang, H., Zhang, L. Q., He, L. Y., and Feng, J. 2009. Genetic diversity of Ralstonia solanacearum strains from China. Eur. J. Plant Pathol. 125:641-653.

Xue, Q. Y., Yin, Y. N., Yang, W., Heuer, H., Prior, P., Guo, J. H., and Smalla, K. 2011. Genetic diversityof Ralstonia solanacearum strains from China assessed by PCR-based fingerprints to unravel host plant- and site-dependent distribution patterns. FEMS Microbiol. Ecol. 75:507-519.

Zheng, X. F., Zhu, Y. J., Liu, B., Zhou, Y., Che, J. M., and Lin, N. Q. 2014 Relationship between Ralstonia solanacearum diversity and severity of bacterial wilt disease in tomato fields in China. J. Phytopathol. 162:607-616. 